\title{
Simulation of Effect of Knife Rollers Rotation Modes on Wind Field
}

\author{
Xu Zhenhua ${ }^{1, a}$, Wang Yuxing ${ }^{1, b^{*}}$, Tang Yanqin ${ }^{1}$, Zhao Feng ${ }^{1}$ \\ ${ }^{1}$ Key Laboratory of Key Technology on Agricultural Machine and Equipment, Ministry of Education, \\ South China Agricultural University, Guangzhou 510642, China \\ a zhenhua090527@163.com, bscauwyx@ scau.edu.cn
}

Key words: Computer simulation; Finite element method; Sugarcane leaf cutting and returning to field machine; Fluid-structure interaction; Wind field

\begin{abstract}
In order to study the influence of different knife rollers rotation modes for the wind field inside the diversion cover of the sugarcane leaf cutting and returning to field machine equipped with flexible cutters, the ALE method of finite element software LS-DYNA was applied to operate the simulation of wind-field fluid-structure interaction inside the diversion cover, which was under the condition of two knife rollers in clockwise rotation and the clockwise rotation of the master knife roller and the anticlockwise rotation of the slave knife roller respectively. According to the comparison of the simulation results, under the condition of two knife rollers in clockwise rotation, the wind field inside the diversion cover was more stable, which would decrease the influence of the wind field on the process of cutting the sugarcane leaves and benefit the output of the sugarcane leaves.
\end{abstract}

\section{Introduction}

One of the important factors that affecting the cutting rate is the wind field inside diversion cover of the sugarcane leaf cutting and returning to field machine equipped with flexible cutters. The different rotation mode of knife rollers has direct influence on the wind field inside diversion cover, so it's an important guarantee to find out how to obtain a more stable wind field in which rotation mode. AS air is invisible object, it's hard to gain the real-time dynamic graph of the wind field inside diversion cover directly through high-speed camera or other devices. Recently, the finite simulation element analysis software LS-DYNA has been widely applied in the fluid-structure interaction simulation of the air. Tutt etc.[1-2]、 Lingard etc. [3-4]、Jia He etc. [5] 、 Cheng Han etc. [6] have made the simulation analysis of the parachute inflation process and the flow field around the parachute based on the ALE method (arbitrary lagrangian-eulerian algorithm). Taylor etc. [7] Ma Chunsheng etc. [8] have developed the fluid-structure interaction simulation of the parachute-capsule system and air in the landing of spacecraft recovery system. Hirth etc. [9]、 $\mathrm{Yu} \mathrm{Li}$ etc. [10] have done the research in numerical simulation of airbag during deploying process. Based on the foundation of the studies mentioned above, the ALE method was used to operate the fluid-structure interaction simulation of the knife rollers rotating in the air, which was to gain the change of wind field in different knife rollers rotation modes. The research results could provide important reference value to a reasonable choice of knife rollers rotation modes. 


\section{Structure of Diversion Cover and Knife Rollers}

The wind field caused by the sugarcane leaf cutting and returning to field machine was generating inside the diversion cover, so the diversion cover and knife rollers of the machine were mainly research subjects in this paper, whose structure was shown in Figure 1. Two knife rollers were assembled on the sugarcane leaf cutting and returning to field machine, and each knife roller was equipped with two cutter disks, on which the flexible cutters made of nylon were installed. Power transmission between driving motor and knife rollers and between the two knife rollers was achieved through belt drive. When machine working, the master knife roller was driven by driving motor through input belt, and the slave knife roller was driven by the master knife roller through output belt, as results of which the flexible nylon cutters would be rotating at high speed to whip and cut the sugarcane leaves. The two knife rollers were installed inside the diversion cover, while the driving motor, input and output belts and belt wheels were installed outside the diversion cover. Wind field caused by the knife rollers was limited inside the diversion cover, which could stop the sugarcane leaves that have been cut being blew away in combination with the pressure from the caterpillar chassis [11-12].


1. Diversion cover
2.Slave knife roller
3.Rack
4.Driving motor
5.Input belt
6. Input belt wheel 7.Master knife roller $\quad 8$.Output belt wheel $\quad 9$. Output belt

Fig.1 Structure sketch of knife roller and diversion cover

\section{Simulation model}

In order to study the effect of different knife rollers rotation modes for the wind field inside diversion cover, two rotation modes were considered in this paper. 1) One rotation mode was that the master knife roller and the slave knife roller were rotating in the same direction, clockwise or anticlockwise, considering the clockwise in this paper, as shown in Figure 2a. 2) The other rotation mode was that the master knife roller was rotating anticlockwise and the slave knife roller was clockwise, as shown in Figure 2b. The rotation speed of master knife roller was $315.15 \mathrm{rad} / \mathrm{s}$, and the rotation speed of slave knife roller was $300.08 \mathrm{rad} / \mathrm{s}$.

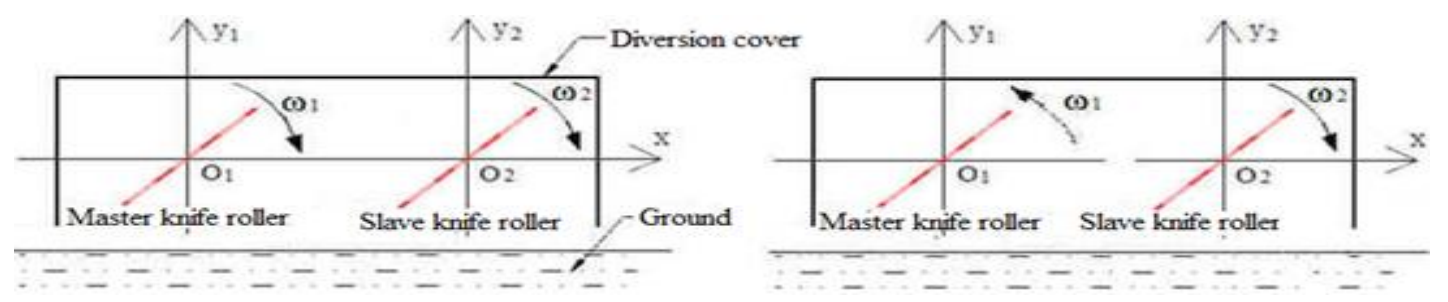

Note: $O_{1}$ and $O_{2}$ are the rotating center of master knife roller and slave knife roller respectively. $x$ is the horizontal axis. $y_{1}$ and $y_{2}$ are the vertical axis of master knife roller and slave knife roller respectively. $\omega_{1}$ is the rotation speed of master knife roller. $\omega_{2}$ is the rotation speed of slave knife roller.
a. Two knife rollers in clockwise rotation
b. Anticlockwise rotation of the master knife roller, clockwise rotation of the slave knife roller

Fig.2 Motion diagram of two different rotation modes 


\section{Simulation Assumptions}

1) Ignore the effect of natural wind outside the diversion cover and assume the model is in a situation of static wind.

2) Ignore the moving displacement of sugarcane leaf cutting and returning to field machine, and assume it's static. The walking velocity of machine is $0.3 \mathrm{~m} / \mathrm{s}$ under working and rotation speed of the knife rollers is $3000 \mathrm{r} / \mathrm{min}$, so the moving displacement of machine would be pretty short during the process of cutting one sugarcane leaf.

3) Assume the ground is flat. The sugarcane field is uneven, which could not be modeled accurately.

4) Because of the tiny deformation, ignore the influence of deformation of ground, knife rollers and diversion cover on wind filed. Set these three parts as rigid body to reduce operation time.

\section{Modeling and Meshing}

When modeling, only the structure inside diversion cover was being considered, while the driving motor and rack outside diversion cover were ignored. Simulation model consist of diversion cover, knife rollers, ground and air. Besides, the knife rollers were composed of rotating shafts, cutter disks and nylon cutters.

The density, elastic modulus and Poisson's ratio of rotating shaft and cutter disk was $7820 \mathrm{~kg} / \mathrm{m}^{3}$, $2.07 \times 10^{11} \mathrm{~Pa}$ and 0.28 , respectively.

The density, elastic modulus and Poisson's ratio of diversion cover was $8000 \mathrm{~kg} / \mathrm{m}^{3}, 2.1 \times 10^{11} \mathrm{~Pa}$ and 0.3 , respectively.

The density, elastic modulus and Poisson's ratio of ground was $1850 \mathrm{~kg} / \mathrm{m}^{3}, 2.0 \times 10^{7} \mathrm{~Pa}$ and 0.3 , respectively.

The material of nylon cutter was PA66, density and shear modulus of which was $1400 \mathrm{~kg} / \mathrm{m}^{3}$ and $3.01 \times 10^{9} \mathrm{~Pa}[13]$ respectively.

Material model of the air was set to *MAT_NULL. Density was $1.25 \mathrm{~kg} / \mathrm{m}^{3}$ and coefficient of kinetic viscosity was $1.7456 \times 10^{-5} \mathrm{~N} \cdot \mathrm{s} / \mathrm{m}^{2}$. Meanwhile, define the linear polynomial equation as *Eos_Linear_Polynomial.

The simulation model was built in ANSYS Graphical User Interface directly with the unit of m-kg-s. The size of air part was slightly bigger than that of diversion cover, therefore air part could contain the whole diversion cover and knife rollers, as shown in Figure 3a. Increase grid density of part of air which was in the nylon knife rotating area. The meshed model was shown in Figure 3b, total node number of which was 141413 and node number of air part was 48546.

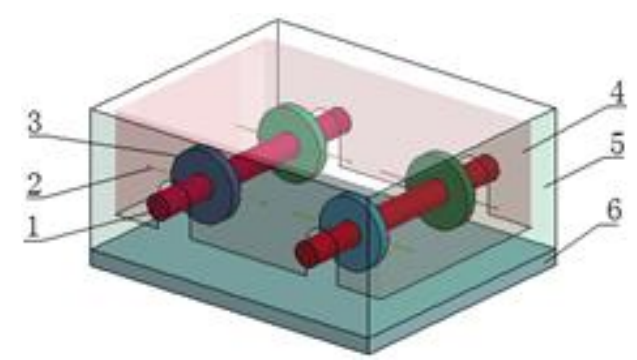

1. Rotating shaft 2. Flexible nylon cutter 3. Cutter disk

4. Diversion cover 5. Air 6. Ground

Fig.3a. unmeshed model

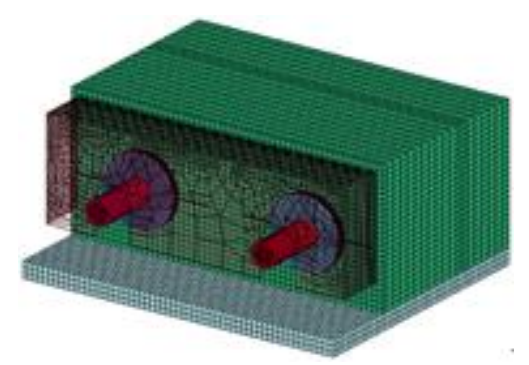

Fig.3b. Cutaway view of meshed model

Fig.3 Simulation model 


\section{Parameter Settings of Fluid-Structure Interaction}

Some important parameter could not be defined in ANSYS Graphical User Interface. Thus, it had to use the LS-PrePost to complete the parameter settings before computing. After modeled and meshed, the K words file was exported from ANSYS Graphical User Interface, and then was added with keywords for fluid-structure interaction control in LS-PrePost. Among them, about the setting of fluid-structure interaction keyword *CONSTRAINED_LAGRANAGE_IN_SOLID, set the air as MASTER, while the knife rollers, diversion cover and ground as SLAVE, and chose the penalty function methods for coupling.

\section{Simulation Operation and Simulation Results Analysis}

\section{K Words File Operation}

After the $\mathrm{K}$ words files modification of two rotation modes of knife rollers were finished in LS-PrePost, they were submitted to LS-DYNA solver for operation, from which simulation results of two modes could be obtained. Based on the simulation results, we could make comparison between two modes in different aspects, such as change of wind field, wind velocity, etc.

\section{Change of Wind Field on Lateral Section of Two Rotation Modes}

The highest wind velocity appeared on the plane which the nylon cutters were rotating, so this plane was chosen to be analyzed.

Figure 4 was, at the time of $0.086 \mathrm{~s}$, the cloud chart and velocity vector chart of wind velocity on the lateral section that nylon cutter were rotating, under the rotation mode of two knife rollers in clockwise rotation. According to the cloud chart of wind velocity, the speed was highest in the area around nylon cutters, and the speed was decreasing quickly in the area where the nylon cutters were leaving. Near the ground, the airflow caused by the slave knife rollers was moving in the left upper direction (as arrow a shown in Figure 4b), and then under the influence of the airflow caused by the master knife rollers (as arrow b shown in Figure 4b), the two airflows were blowing out from the outlet of diversion cover together, as arrow c shown in Figure 5b. What' more, an airflow convergence surface (as curve d shown in Figure 4b) was generating during the process of the airflow interaction caused by two rotary knife rollers, which was accompanied by the generation of one or two vortexes.

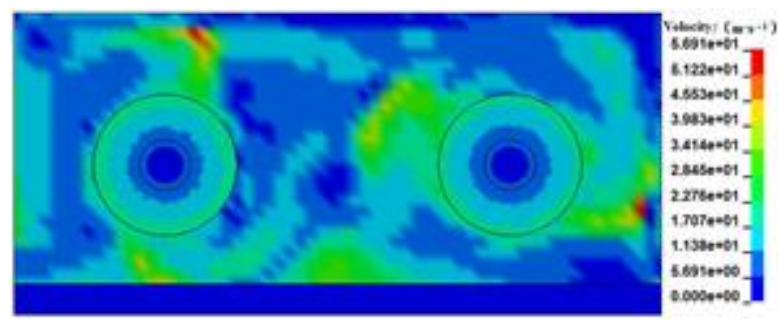

a. Cloud chart

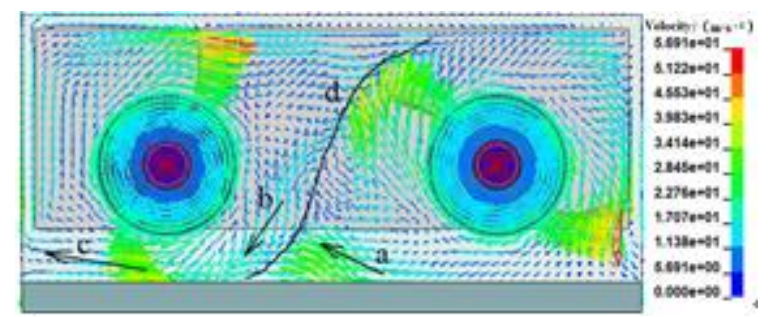

b. Velocity vector

Fig.4 Lateral section wind field of two knife rollers in clockwise rotation

Figure 5 was, at the time of $0.034 \mathrm{~s}$, the cloud chart and velocity vector chart of wind velocity on the lateral section that nylon cutters were rotating, under the rotation mode of anticlockwise rotation of the master knife roller and clockwise rotation of the slave knife roller. From the cloud chart of wind velocity, the speed was highest in the area around nylon cutters and middle area of diversion 
cover. In Figure 5b, in the middle area of diversion cover, the moving direction of airflow caused by the master knife roller was as the arrow a shown, and the moving direction of airflow caused by the slave knife roller was as the arrow b shown, both of whose velocity were close. After the convergence of the two airflows, the direction of airflow caused by the master knife roller would be changed, as the arrow c shown, so did the airflow caused by the slave knife roller, as the arrow $\mathrm{d}$ shown. The two airflows were converging and moving toward the top area of diversion cover, the velocity of which was getting higher.

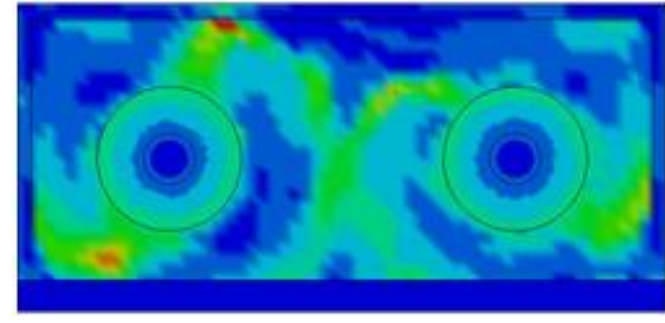

a. Cloud chart

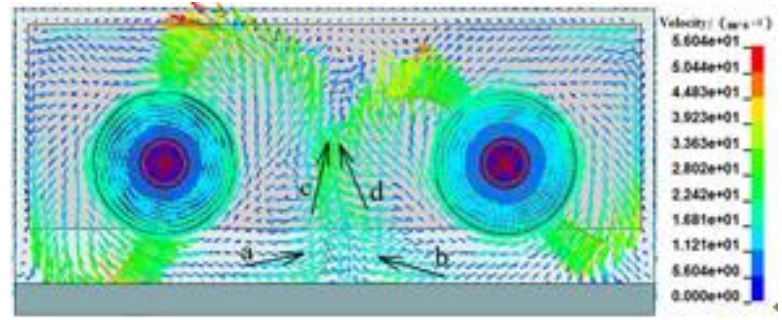

b. Velocity vector

Fig.5 Lateral section wind field of anticlockwise rotation of the master knife roller, clockwise rotation of the slave knife roller

\section{Change of Wind Field on Cross Section of Two Rotation Modes}

Figure 6 was, at the time of $0.082 \mathrm{~s}$, the cloud chart and velocity vector chart of wind velocity on the vertical cross-sectional plane between the two knife rollers, under the rotation mode of two knife rollers in clockwise rotation. From the cloud chart, it could be seen that the wind speed was higher in the area where the nylon cutters were rotating, while the wind speed was low between two cutter disks on the same knife rollers. Air movement caused by the rotation of the rollers was mainly within its cross-section, and the intersection of air flow was not strong in the longitudinal direction. There were eddy currents close to nylon cutters rotating field.

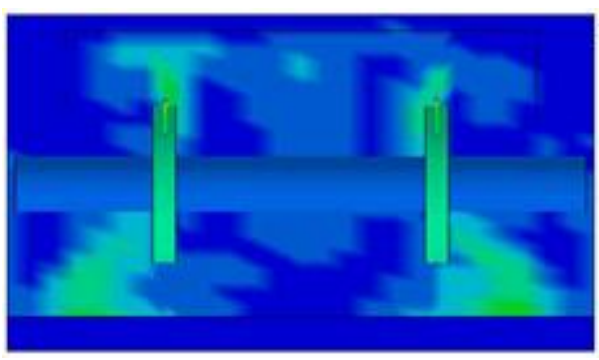

a. Cloud chart

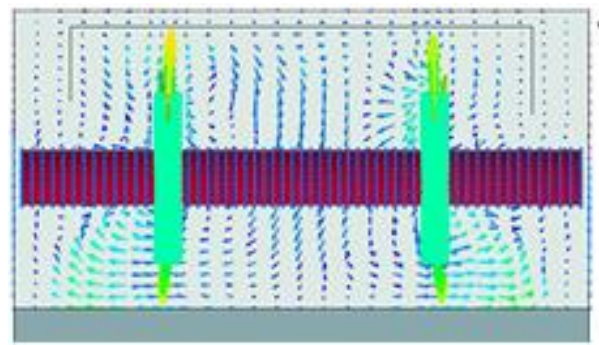

b. Velocity vector

Fig.6 Cross section wind field of two knife rollers in clockwise rotation

Figure 7 was, at the time of $0.052 \mathrm{~s}$, the cloud chart and velocity vector chart of wind velocity on the vertical cross-sectional plane between the two knife rollers, under the rotation mode of anticlockwise rotation of the master knife roller and clockwise rotation of the slave knife roller. In this operating mode, the airflow converged in the middle of diversion cover. After the intersection of airflow in the longitudinal direction, it would move in diffusion on both sides. Wind speed between the two cutter disks on the same knife roller was obviously higher than the wind speed of two knife rollers in clockwise rotation. The upward movement of air would rebound and reflux on the top of diversion cover, which was easy to form a vortex in that area. 


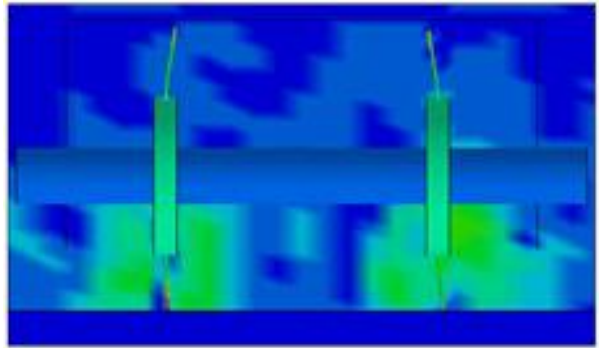

a. Cloud chart

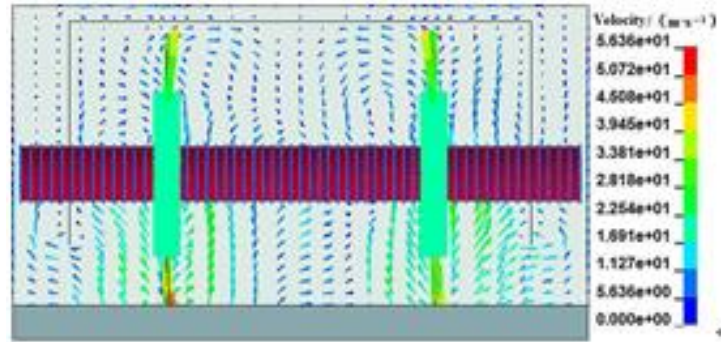

b. Velocity vector

Fig.7 Cross section wind field of anticlockwise rotation of the master knife roller, clockwise rotation of the slave knife roller

\section{Comparison of Wind Velocity of Different Location inside Diversion Cover}

The average wind speed at different locations inside diversion cover within two working mode simulation results were compared, extract nodes from three main regions in the plane the nylon cutters rotating (Figure 8), respectively, the left outlet of diversion cover (in the area shown in Figure a), region between the two knife rollers (in the region shown in Figure B), right outlet of diversion cover (in the area shown in Figure C). In the area of left and right outlet of diversion cover, started to select the node every $0.01 \mathrm{~m}$, from the height of $0.01 \mathrm{~m}$ above ground, totally 5 nodes. But, in the area between two knife rollers, started to select the node every $0.02 \mathrm{~m}$, from the height of $0.02 \mathrm{~m}$ above ground, totally 12 nodes.

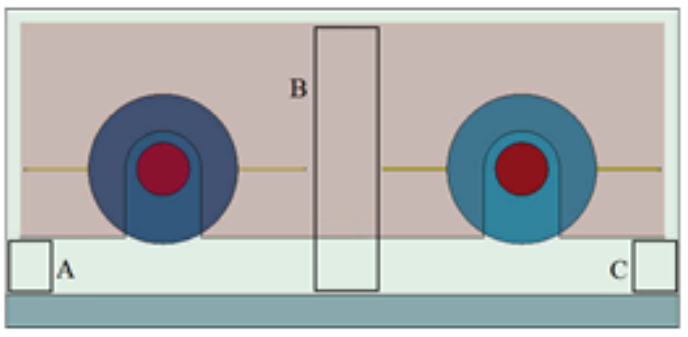

Fig.8 Areas of selected nodes

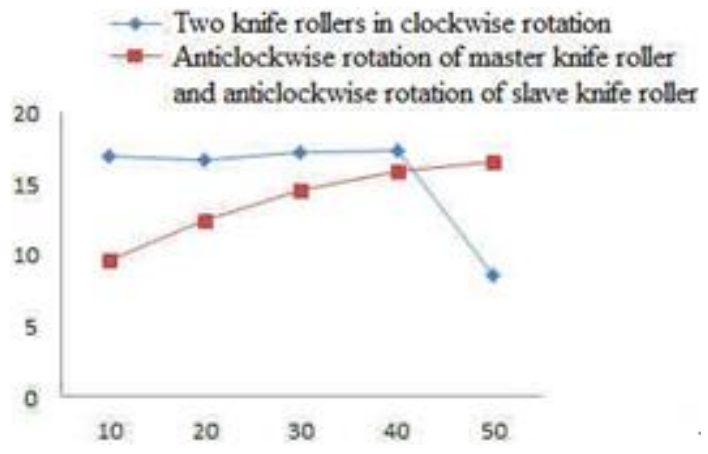

Fig.9 Comparison of wind velocity in the left outlet of diversion cover

Figure 9, 10 and 11 were comparison of wind velocity between two modes within three working area. As shown in Figure 9, in the left outlet of diversion cover, wind speed were more stable under the condition of two knife rollers in clockwise rotation, but at $0.05 \mathrm{~m}$ height (diversion cover bottom edge) wind speed was significantly reduced; Under condition of master knife roller in anticlockwise rotation and slave roller in clockwise rotation, wind speed was showing rising trend, and the wind speed is lower than that of two knife rollers in clockwise rotation from an overall perspective.

As shown in Figure 10, in the area between the two knife rollers, under the condition of two knife rollers in clockwise rotation, wind speed was decreasing with height increasing, then remained at a relatively stable value, which was due to presence of airflow convergence surface and wind speed was less volatile; Under condition of anticlockwise rotation of the master knife roller 
and anticlockwise rotation of the slave knife roller, wind speed was increasing firstly then decreasing; The reason was that, at the height between 0 and $0.06 \mathrm{~m}$, airflow began intersection, as result of which wind speed increased with altitude increased and then during upward movement of the convergent airflow, airflow intensity began to decay and wind speed began to drop. The wind speed was higher than the value of the two knife rollers in clockwise rotation.

As shown in Figure 11, in the right outlet of diversion cover, wind speed and changing trend of wind field of the two rotation modes was almost the same.

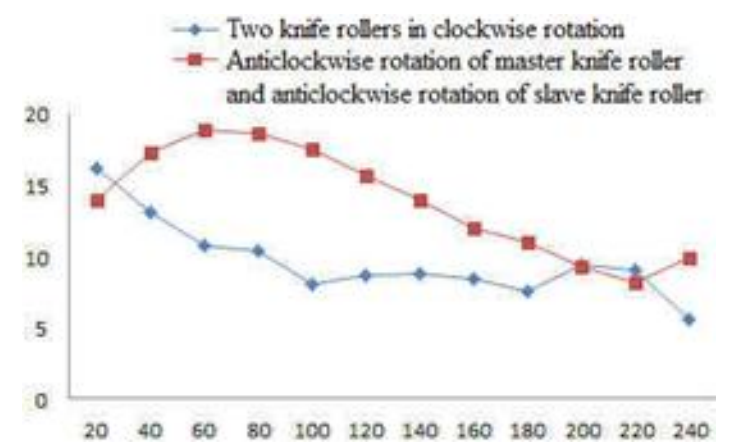

Fig.10 Comparison of wind velocity in area between the knife rollers

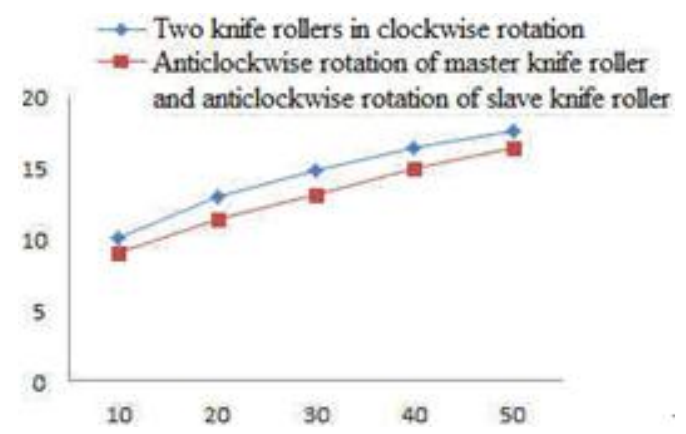

Fig.11 Comparison of wind velocity in the right outlet of diversion cover

\section{Conclusions}

Through the simulation of the two conditions, the wind changes inside the diversion cover under two conditions were obtained, the simulation results show that:

1) Under the rotation mode of two knife rollers in clockwise rotation, from the analysis of wind field on lateral section, an airflow convergence surface was generating in the middle area between two knife rollers, which was accompanied by the generation of one or two vortexes. And from the analysis of wind field on cross-sectional plane, Air movement caused by the rotation of the rollers was mainly within its cross-section, and the intersection of air flow was not strong in the longitudinal direction. Under the rotation mode of clockwise rotation of the master knife roller and anticlockwise rotation of the slave knife roller, the two airflows were converging and moving toward the top area of diversion cover, the velocity of which was getting higher. On the cross-sectional plane, after the intersection of airflow in the longitudinal direction, it would move in diffusion on both sides. The upward movement of air would rebound and reflux on the top of diversion cover, which was easy to form a vortex in that area.

2) On the comparison of the wind velocity between the rotation modes, the velocity of the two knife rollers in clockwise rotation was stable in the middle area of two knife rollers and the left outlet of diversion cover, which benefited the emission of sugarcane leaves after being cut. As for the rotation mode of clockwise rotation of the master knife roller and anticlockwise rotation of the slave knife roller, there was an obvious changing trend of wind speed in that three areas. Moreover, under this rotation mode, the airflow was moving upward with high speed, which was easy to blow the sugarcane leaves in the air and leading to sugarcane leaves wrapped around the knife roller, resulting in subsequent cutting rate.

3) The rotation of two knife rollers in clockwise rotation could form a more stable flow field and reduce the impact of the self-excited wind farm on sugarcane leaves cutting. 


\section{Acknowledgements}

This work was financially supported by Doctoral Fund of Ministry of Education of China (No. 20124404110002). Corresponding author: Wang Yuxing.

\section{References}

[1]. Tutt B A, Taylor A P. The use of LS-DYNA to simulate the inflation of a parachute canopy[R].AIAA 2005-1608, 2005.

[2]. Tutt B A. The application of a new material porosity algorithm for parachute analysis[C]// The $9^{\text {th }}$ International LS-DYNA Users Conference, 2006.

[3]. Lingard J S, Darley M G. Simulation of parachute fluid structure interaction in supersonic flow[R].AIAA 2005-1607, 2005.

[4]. Lingard J S, Darley M G, Underwood J C. Simulation of mars supersonic parachute performance and dynamics[R]. AIAA 2007-2507, 2007.

[5]. Jia He, Rong Wei, Chen Guoliang, et al. The simulation of parachute inflation process based on LS-DYNA software [J]. Spacecraft Environment Engineering, 2010，27(3): 367-373.

[6]. Chen Han, Yu Li, Li Shengquan. Numerical simulation of parachute inflation process based on ALE [J]. Journal of Nanjing University of Aeronautics\& Astronautics, 2012, 44(3): 290-293.

[7]. Taylor A P. Developments in the application of LS-DYNA to fluid structure interaction (FSI) problems in recovery system design and analysis [C] // The 7th International LS-DYNA Users Conference, Fluid /Structure. Michigan: LSTC \& ETA, 2002: 10-17.

[8]. Ma Chunsheng, Huang Shilin, Zhang Jinhuan, et al. Simulations of the landing of manned spacecraft with parachutes using the ALE method in LS-DYNA[J].Journal of Tsinghua University Science and Technology, 2006, 46(8): 1455-1457.

[9]. Hirth A, Haufe A, Olovsson L. Airbag simulation with LS-DYNA past-present-future [C] // Frankenthal: 6th European LS-DYNA User's Conference, 2007

[10]. Yu Li, Chen Han, Liu Xiong. Numerical simulation of airbag during deploying process [J]. Journal of Nanjing University of Aeronautics\& Astronautics, 2010，42(4): 472-476.

[11]. Wang Jing. Design and Research of Sugarcane Leaf Cutting off Returning to Field Machinery[D].Guangzhou: South China Agricultural University, 2014.

[12]. Jing Wang, Yuxing Wang, Yanqin Tang, et al. Finite element analysis of the chassis for sugarcane leaf cutting off returning to field machinery [J]. Advanced Materials Research, 2014, 915-916: 305-309.

[13]. Jiaxian Lu, Yuxing Wang, Yanqin Tang, et al. Fatigue analysis of large-deformation cutting tool used in cutting-off sugarcane-leaf returning machine [J]. Researches and Applications in Mechanical Engineering, Volume 3, 2014: 54-57. 